\title{
Anabases
}

ANABASES Traditions et réceptions de l'Antiquité

$9 \mid 2009$

Varia

\section{Annemarie Schwarzenbach et l'Orient : interview avec Dominique Laure Miermont et Nicole Le Bris}

Dominique Laure Miermont et Nicole Le Bris

\section{(2) OpenEdition}

\section{Journals}

Édition électronique

URL : http://journals.openedition.org/anabases/534

DOI : 10.4000/anabases.534

ISSN : 2256-9421

Éditeur

E.R.A.S.M.E.

\section{Édition imprimée}

Date de publication : 1 mars 2009

Pagination : 285-296

ISSN : 1774-4296

\section{Référence électronique}

Dominique Laure Miermont et Nicole Le Bris, « Annemarie Schwarzenbach et l'Orient : interview avec

Dominique Laure Miermont et Nicole Le Bris", Anabases [En ligne], 9 | 2009, mis en ligne le 01 mars

2012, consulté le 21 octobre 2019. URL : http://journals.openedition.org/anabases/534 ; DOI :

10.4000 /anabases.534

Ce document a été généré automatiquement le 21 octobre 2019

(c) Anabases 


\title{
Annemarie Schwarzenbach et l'Orient : interview avec Dominique Laure Miermont et Nicole Le Bris
}

\author{
Dominique Laure Miermont et Nicole Le Bris
}

\begin{abstract}
A : Dominique Laure Miermont, vous avez publié aux éditions Payot une biographie intitulée Annemarie Schwarzenbach ou le mal d'Europe. Pouvez-vous évoquer pour nous sa vie et sa personnalité ?
\end{abstract}

DLM : Annemarie Schwarzenbach (AS) est née à Zurich en 1908, dans une famille de la haute bourgeoisie helvétique. Brillante élève, elle passa l'examen de "maturité » en 1927, fit des études d'histoire et obtint le titre de «Dr.phil.» (équivalent de l'actuel magister) en 1931.

Se vouant dès lors à l'écriture, elle s'installa quelque temps à Berlin (1931-33). Dans le sillage de Klaus et Erika Mann, avec qui elle se lia d'amitié à partir de 1930, elle prit position contre le national-socialisme et initia la revue antifasciste Die Sammlung ("Le Rassemblement").

Par ailleurs, elle entama dès 1930 une carrière de journaliste et devint, à partir de 1933, reporter-photographe, ce qui la conduisit dans différents pays européens, au Proche et au Moyen-Orient, en Amérique du Nord et en Afrique (Congo, Maroc). Très appréciée des rédactions, elle publia en une dizaine d'années près de 300 articles dans la presse helvétique. Le fonds portant son nom aux archives de Berne compte environ 5000 négatifs et tirages originaux.

Sur le plan personnel, AS était un être tourmenté, en proie à un mal de vivre qu'elle tenta de juguler par la consommation de diverses drogues (alcool, tabac, produits morphiniques) auxquelles son organisme ne put résister plus de dix ans. Elle mourut en 1942, à l'âge de 34 ans, des suites d'une chute de vélo très probablement causée par un état de délabrement physique et psychique avancé.

AS a promené sur le monde un regard empreint d'humanité et d'humanisme. Elle avait une conscience aiguë du tragique de la condition humaine, une connaissance intime de l'ambiguïté des choses de ce monde, une perception infaillible des mécanismes pernicieux des idéologies. Ses idéaux de paix, de liberté individuelle et 
de justice la rendirent très proche des déshérités et des laissés-pour-compte de tous pays. Elle était douée d'une empathie pour ses «frères humains " qui s'exprime à travers l'écriture et la photographie. C'était aussi une femme animée d'un authentique courage subversif, aux antipodes de ses fragilités.

Outre sept livres publiés de son vivant, AS écrivit de nombreux autres ouvrages dont une partie a été éditée depuis sa " renaissance » en 1987. Son œuvre est considérable et protéiforme : romans, guides touristiques, nouvelles, journaux de voyage, lettres, biographie, pièce de théâtre, poèmes, ainsi que de nombreux textes inclassables.

A : Comment Annemarie Schwarzenbach a-t-elle été conduite à s'occuper d'archéologie ?

DLM : On peut supposer que, du fait de ses études d'histoire, elle a été en contact avec des archéologues. Ce qui est certain, c'est qu'on lui a proposé en juin/juillet 1933 de se joindre à un groupe dont le projet était de partir pendant six mois pour visiter une dizaine de champs de fouilles situés entre la Turquie et la Perse.

NLB : L'archéologie apparaît comme un complément et un prolongement de sa formation d'historienne. Mais à lire ses lettres, ce qui détermine son départ, c'est un ensemble de raisons, dont les premières sont psychologiques et morales. Elle est en quête de santé morale. Ses rapports avec sa famille sont difficiles - et cela de plus en plus, à mesure que s'affermissent des prises de position radicalement opposées, prohitlériennes dans sa famille, résolument anti-nazies chez elle. À 25 ans il lui faut secouer sa dépendance à l'égard de sa famille, lui prouver qu'elle n'est pas « incapable » et « dilettante ». Elle tirera profit, pense-t-elle, d'un travail « concret » et " objectif ${ }^{1}$ » (par opposition sans doute aux travaux littéraires auxquels est allée jusqu'alors sa prédilection, travaux qui la maintenaient dans un contact trop exclusif avec sa subjectivité). Il sera salutaire aussi qu'elle s'éloigne de ses amis - Erika et Klaus Mann en particulier - auxquels elle se sent liée par une excessive dépendance affective.

D'autres raisons ont puissamment joué, qui sont d'ordre intellectuel et finalement politique. Annemarie a été attentive à la façon dont, depuis les années 20 , on utilise abondamment à des fins idéologiques les racines antiques de la civilisation occidentale. Elle a lu (en 1930) l'ouvrage du maurassien Henri Massis, Défense de l'Occident (1927) ${ }^{2}$, ou encore Le Déclin de l'Occident d'Oswald Spengler (1922) ${ }^{3}$. Maintenant que le nazisme déferle, en 1933-34 elle sent le besoin d'échapper, pour mieux le combattre, à son emprise paralysante ${ }^{4}$. Les formes d'engagement qui se proposent en Europe ne la satisfont pas. Celle qu'elle choisit est double : fonder avec Klaus Mann la revue antifasciste que nous disions et, paradoxalement, partir en Orient faire des fouilles archéologiques: elle pourra, comme elle l'écrit à Klaus $M{ }^{5} n^{5}$, en mesurant des crânes, "prouver l'absurdité de ces idiots de racistes allemands ", c'est-à-dire l'absurdité des théories sur la supériorité de la race aryenne...

A : Comment s'est-elle formée à l'archéologie et quels sites a-t-elle visités ?

DLM : Avant de partir, elle a lu nombre d'ouvrages spécialisés. Et déjà en 1932, projetant un voyage en Perse, qui avorta au dernier moment, elle avait étudié les collections asiatiques des musées de Berlin. Au cours de ce séjour de six mois - entre octobre 1933 et avril 1934 -, c'est sur le chantier syrien de Reyhanli (à mi-distance entre Antakya et Alep), dirigé par la mission américaine de l'université de Chicago, qu'elle s'initie pendant plus d'un mois aux rudiments de l'archéologie. Ensuite, elle visite de nombreux sites sur le sol de l'ancienne Mésopotamie: Ctésiphon, Tello, 
Uruk, Qal'at Sukkar. À Babylone, Ur et Khafadjé, ce sont respectivement les célèbres professeurs Jordan, Woolley et Frankfort qui l'accueillent. En Iran, après Suse, AS visite le chantier de Rayy (ancienne Rhagès), près de Téhéran. Le professeur Erich Schmidt, qui en est le directeur pour le compte de l'University Museum de Philadelphie et du Museum of Fine Arts de Boston, lui propose alors de revenir en octobre de cette même année 1934 participer aux fouilles - ce qu'elle accepte aussitôt. Enfin, ce périple se termine sur le site de Persépolis où elle rencontre Friedrich Krefter, le fameux archéologue dont les croquis et les plans extraordinairement précis de l'ancienne capitale achéménide ont permis de réaliser en 2006 une reconstitution du site en trois dimensions. Et c'est lui en personne qui photographie AS sur le champ de fouilles (figure 1).

Figure 1. Sur les fouilles de Persépolis (1934). Photographie prise par Friedrich Krefter. Fonds D.L. Miermont, Paris.Note manuscrite d'AS au dos : « ich grabe aus » (" je fouille »)

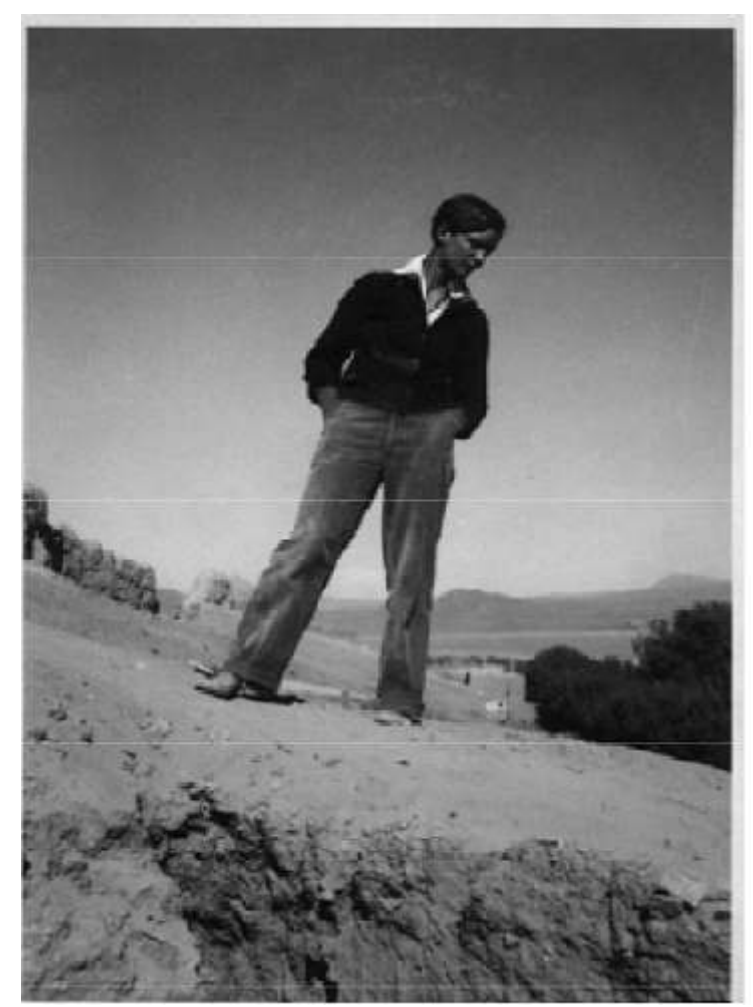

A : Qu'a représenté pour Annemarie Schwarzenbach la pratique de l'archéologie?

NLB : Son expérience d'archéologue se retrouve au travers d'allusions dans les nouvelles d'Orient Exils, mais elle se reflète essentiellement dans deux de ses ouvrages : son récit de voyage Hiver au Proche-Orient (publié dès 1934), et le roman La Vallée Heureuse (commencé en octobre 1938 et paru en 1940), dont le héros est précisément un archéologue. Ce dernier texte permet d'imaginer la façon dont elle a vécu ses campagnes de fouilles. Une indication retient l'attention: le personnage évoque les quelques livres qu'il a choisi d'emporter: "Un gros volume, Cambridge Ancient History; un petit livre avec une couverture rouge, Pottery of the Near East, publié par le British Museum; les lettres de Diotima à Hölderlin, La Résurrection des villes mortes de Marcel Brion et un roman anglais que je n'ai pas $1 u^{6}$.» Les deux tomes du livre de Brion ont été publiés entre décembre 1937 et mai 1938, et n'ont donc pu servir de mentor à l'apprentie archéologue qu'a été AS, mais elle a dû y rencontrer 
après coup une vision du passé et de la découverte archéologique qui correspondait à la sienne. Et de fait on voit sans mal dans l'ouvrage de Brion ce qui a pu faire écho à sa manière de sentir : certaines évocations épiques et lyriques des grands désastres qui s'abattent sur l'humanité ; l'émotion devant une science qui rend vivant, actuel et présent le tragique du passé ; et qui donne le sentiment d'une unité de l'aventure humaine, en rétablissant le lien entre l'homme d'aujourd'hui et l'homme des temps les plus reculés; ou encore cette vision de la marche de l'histoire comme un perpétuel mouvement, un voyage des civilisations, producteur de rencontres infiniment fécondes - Marcel Brion et Annemarie Schwarzenbach ont été tous deux de grands voyageurs.

$\mathrm{Au}$ total, AS s'est indéniablement beaucoup investie dans son expérience d'archéologue (figure 2). Elle s'y est longuement préparée à Berlin, elle a appris l'arabe et le persan. Ses lettres ou ses récits révèlent la passion que peut lui inspirer, sur un site, une hypothèse nouvelle; l'émotion qu'elle éprouve à se trouver sur les lieux mêmes où fut inventée l'écriture, à retrouver l'espace des origines, à « descendre jusqu'à la source la plus profonde ${ }^{7}$ »; et l'intensité de sa rencontre avec certains monuments, qui engage l'idée même qu'elle se fait de l'humanité ${ }^{8}$.

Figure 2. À Farmanieh, près de Téhéran (1935). Photographie prise par Claude Achille Clarac

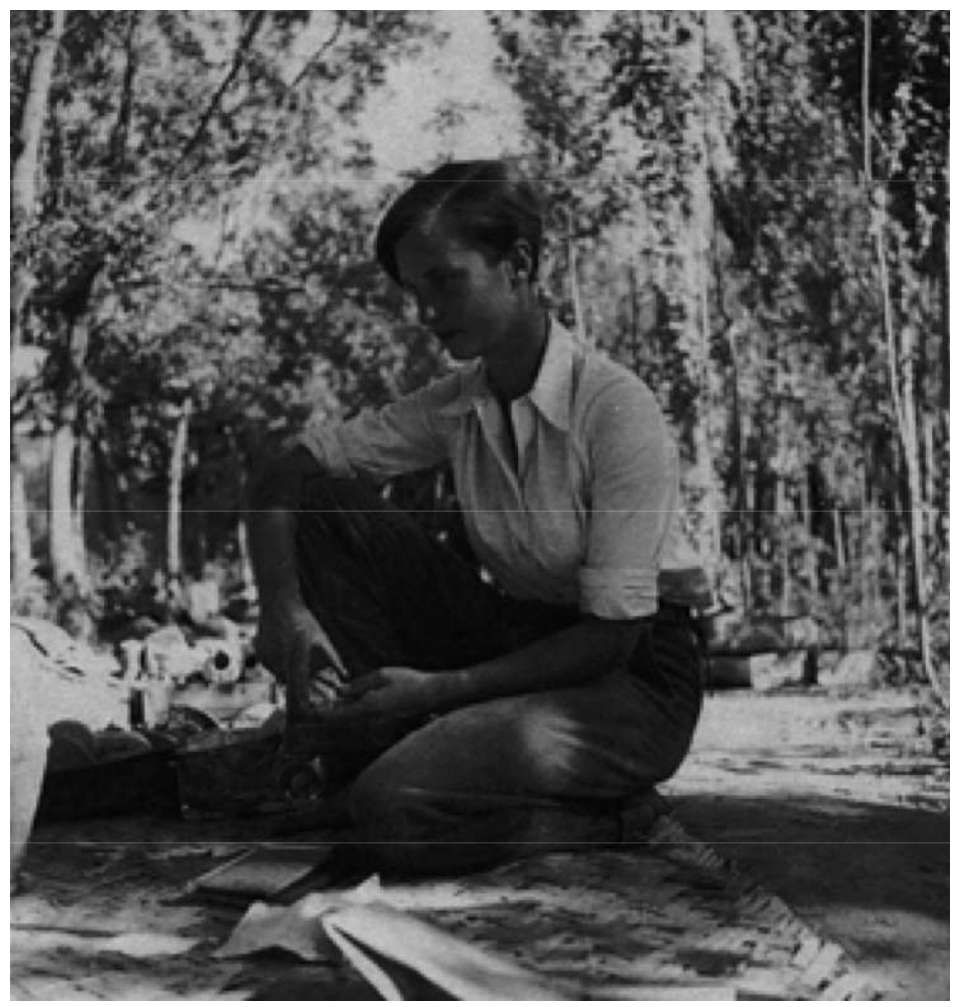

Fonds Henri Pagau-Clarac, Oudon

Cela dit, elle n'a été archéologue qu'un court laps de temps, et toujours, se reprochet-elle, trop en "dilettante». L'essentiel a toujours été pour elle d'écrire; elle a d'ailleurs continué, pendant les fouilles de 1933, à rédiger des reportages et à travailler à son journal de voyage. Et elle dit par la voix du héros de La Vallée Heureuse l'impossibilité pour elle d'avoir un métier à demeure, de développer des racines, et l'invincible besoin de toujours repartir. 
A : Vous faisiez tout à l'heure le lien entre le goût pour l'archéologie et le goût du voyage chez Annemarie Schwarzenbach. Quels ont été ses principaux voyages, et quelles raisons l'ont poussée à les entreprendre?

DLM : Nous avons vu pour quelles raisons AS a effectué ses deux premiers séjours au Proche-Orient. Il y eut ensuite, en 1935, un troisième séjour en Perse, motivé par son mariage avec le diplomate français Claude Achille Clarac, second secrétaire à la légation de France à Téhéran. Enfin, entre juin et août 1939, AS fit en voiture, et en compagnie d'Ella Maillart ${ }^{9}$, le trajet Genève-Kaboul. Après une année passée en cures de désintoxication répétées, ce qui motive ce voyage, c'est surtout le besoin pressant de trouver une activité qui l'éloigne d'elle-même. Grâce à Ella Maillart qui connaît bien Joseph et Ria Hackin ${ }^{10}$, elle a la possibilité de travailler pour la DAFA (Délégation Archéologique Française en Afghanistan). C'est ainsi qu'elle visitera les fouilles de Bagram et travaillera quelque temps sur le site de Konduz (Turkestan afghan) (figure 3).

Entre 1936 et 1938, AS a également fait deux séjours aux USA pour enquêter dans l'Amérique de la Grande Dépression, d'abord dans la région minière de Pittsburgh (Virginie occidentale et Pennsylvanie), ensuite dans les États du Sud (Tennessee, Alabama, Géorgie, Caroline, Ohio). Farouche adepte du New Deal de Roosevelt, elle a réalisé de nombreux reportages dans lesquels elle exprime sa solidarité avec le mouvement syndical naissant et s'indigne des conditions de vie inhumaines auxquelles sont réduits les métayers et les ouvriers des filatures.

Son dernier grand voyage, ce fut le Congo en 1941-42. Elle y partit dans le but de rejoindre les Forces françaises libres et de travailler comme correspondante de guerre. Ce projet échoua, car son amitié avec Klaus et Erika Mann, en lutte contre Hitler, et sa qualité de femme d'un diplomate encore attaché au gouvernement de Vichy $^{11}$, la rendirent suspecte aux yeux des autorités locales. Mais elle y passa dix mois à explorer plusieurs régions, et surtout à écrire.

NLB : On le voit, à l'origine de ces voyages apparaît, presque à chaque fois, une raison professionnelle, ou un désir d'engagement. Mais par delà ces raisons circonstancielles, et sans doute de façon beaucoup plus déterminante, joue le besoin de partir en lui-même, la fascination pour le lointain. C'est ce que disent abondamment des textes comme La Vallée Heureuse et Les Quarante Colonnes du souvenir.

Cela dit, AS n'est pas exactement l'« écrivain voyageur » qu'on voit souvent en elle, sans doute par rapprochement avec Ella Maillart, ou avec Nicolas Bouvier qui reprit les mêmes routes, ou simplement parce qu'on s'arrête à ses travaux de reporter. Elle n'a pas le goût de l'aventure pour elle-même, ni le goût sportif de l'exploit ou de l'épreuve à surmonter. Elle a peu de curiosité ethnographique, et le déclare nettement par la voix du héros de La Vallée Heureuse: "Je ne voyage pas pour découvrir de nouvelles vertus et d'autres mœurs ${ }^{12}$. " Les diverses façons de vivre des hommes l'intéressent moins que leur communauté de destin. Et son propos n'est pas d'écrire pour raconter les péripéties d'un trajet ou les étonnements qu'il procure. 
Figure 3. Annemarie Schwarzenbach avec Ria Hackin, Jacques Meunié et Joseph Hackin de la DAFA, lors d'un pique-nique, Kaboul, automne 1939. Photographie prise par Ella Maillart

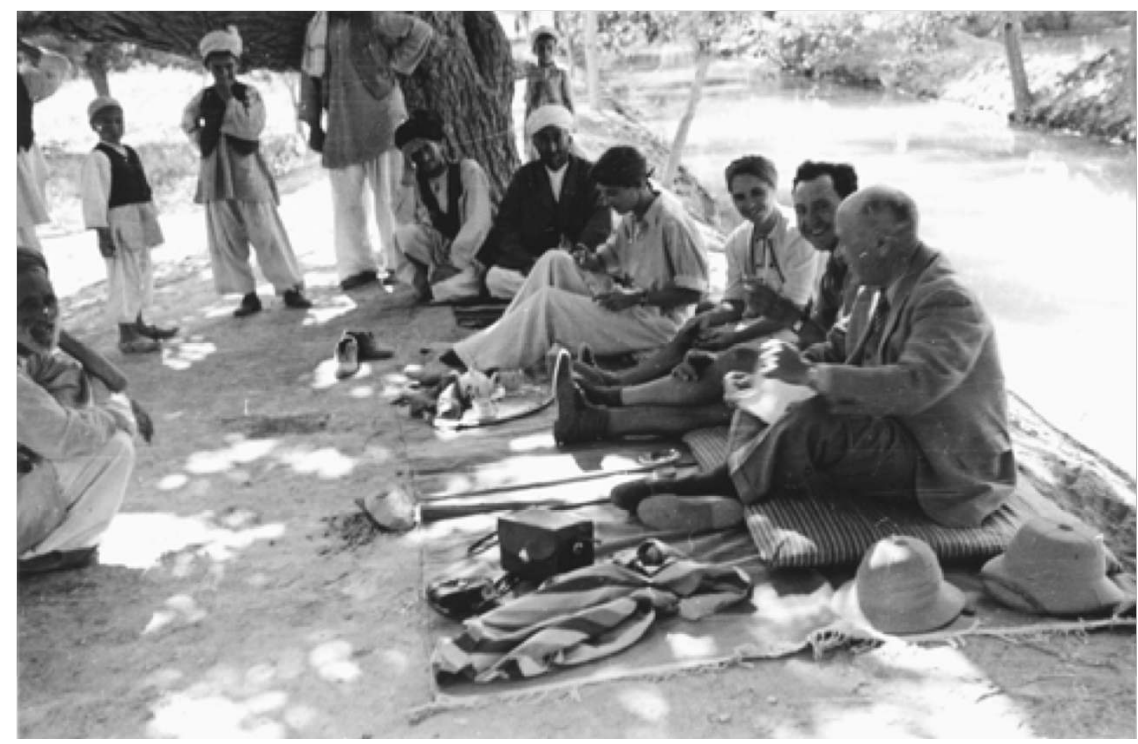

Fonds Ella Maillart au Musée de l'Elysée de Lausanne

Si elle écrit, c'est en dernier ressort surtout pour partager avec ses lecteurs une expérience plus profonde et plus mystérieuse, à caractère spirituel. Je dis mystérieuse parce qu'elle-même déclare souvent ne pas comprendre exactement chez elle ce qu'elle appelle « la malédiction de la fuite »; comme si voyager signifiait céder à une tentation obscure. Une tentation blâmable aussi : comme d'autres écrivains et artistes de ce temps, elle revient souvent à la parabole du Fils Prodigue, auquel elle doit s'identifier quelque peu, avec la dose de culpabilité que cela comporte. C'est seulement peu à peu, au fil du temps, qu'elle se convainc de la validité de sa vocation de voyageuse. Il lui apparaît alors qu'au long de ses voyages, c'est une vision de la condition humaine qu'elle tente, qu'elle a mission, d'acquérir et d'exprimer. Et le voyage même, tel qu'elle l'éprouve, figure le tragique de cette condition, parce qu'il apparaît en dernière analyse comme la quête, toujours déçue et jamais renoncée, d'une plénitude perdue : Où est la terre des promesses ?, tel est le titre parlant qu'on a pu donner à un recueil de ses articles. La famille de pensée à laquelle AS appartient, bien plus que celle des écrivains voyageurs, c'est celle des poètes romantiques, d'Hölderlin et de Rilke.

\section{Ouvrages d'Annemarie Schwarzenbach parus en traduction française (chronologie historique)}

Les dates entre crochets sont les dates d'écriture.

Deux dates séparées par un point-virgule correspondent à l'écriture et à la publication. Les astérisques désignent les ouvrages publiés du vivant de l'auteur.

\section{Guvres littéraires}

Nouvelle parisienne [1929], in Inverses $n^{\circ} 6,2006$

Voir une femme [1929], Métropolis 2008

*Nouvelle lyrique [1931;1933], Verdier 1994

*Hiver au Proche-Orient [1934], Payot 2006

Le Refuge des cimes [1933], Payot 2004

Orient exils [1934-35], Autrement 1994/Payot 2000, 2003

La Mort en Perse [1935-36], Payot 1997, 1998, 2001 
*La Vallée Heureuse [1938 ; 1940], Éditions de l'Aire 1991/ L’Aire bleue 2001

Les Quarante Colonnes du souvenir [1939-40], esperluète éditions 2008

Rives du Congo / Tétouan [1941-42], esperluète éditions 2005

\section{Reportages et correspondance}

Lettres à Claude Bourdet. 1931-1938, Zoé 2008

Loin de New York. Reportages et photographies [1936-38], Payot 2000

Où est la terre des promesses? Avec Ella Maillart en Afghanistan [1939-40], Payot 2002

Visions d'Afghanistan [1939-40], Payot 2002 [hors commerce]

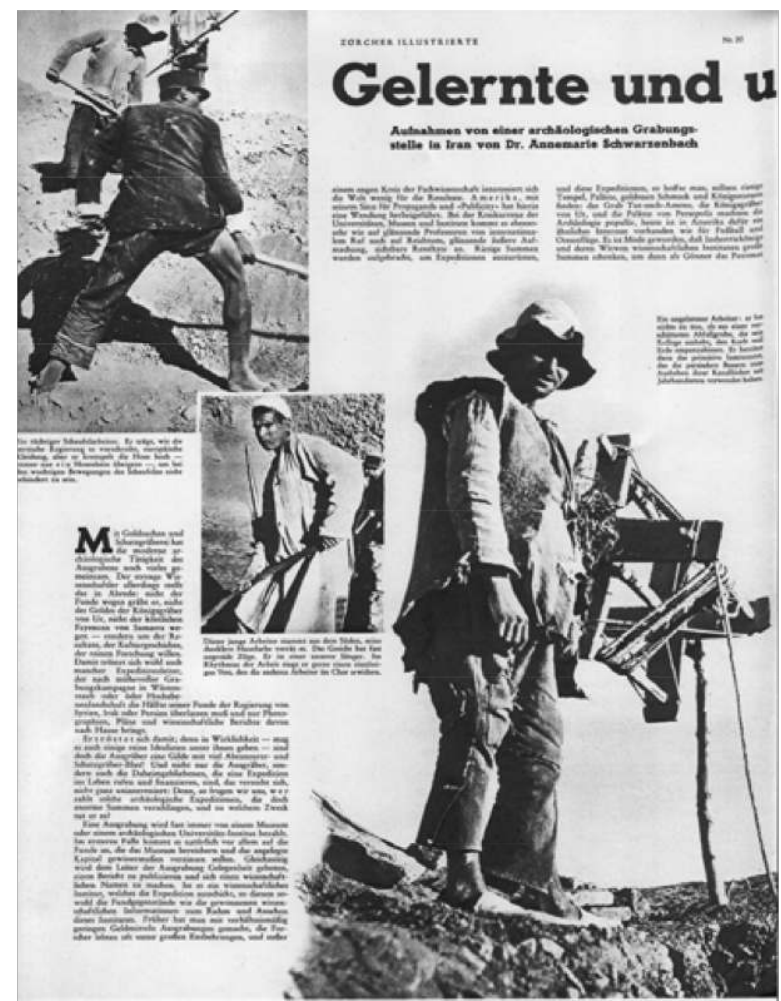




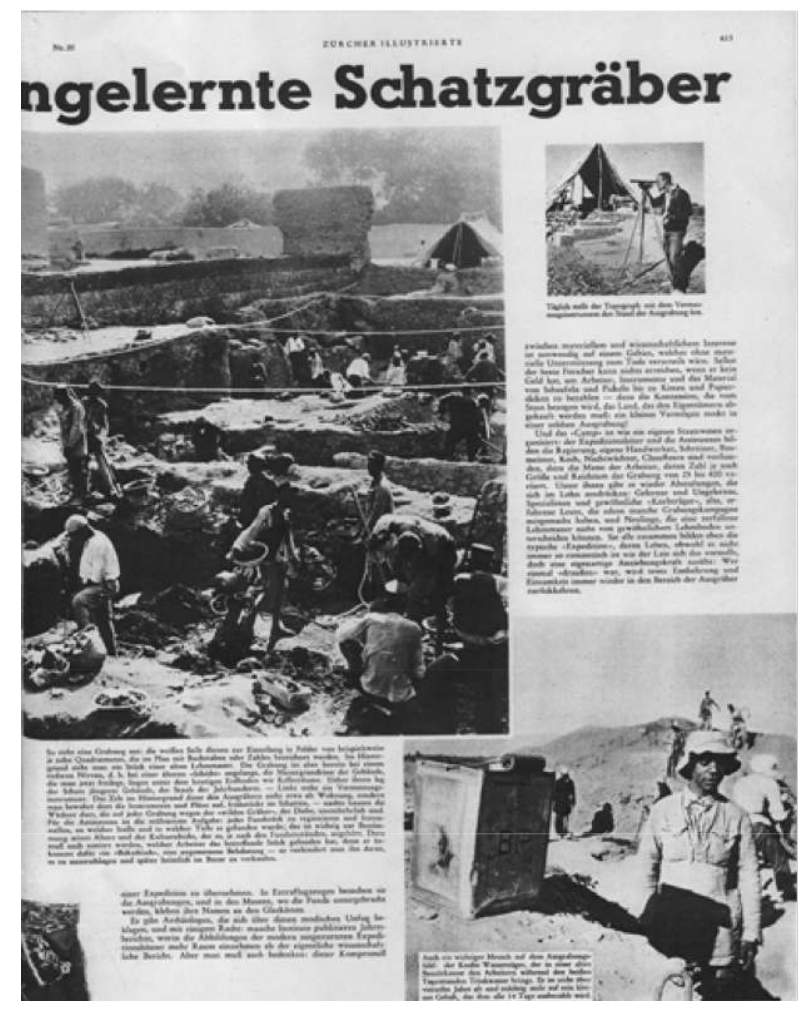

"Fouilleurs qualifiés et non qualifiés". Reportage paru dans le Zürcher Illustrierte du 17 mai 1935, traduit par D.L. Miermont. Photographies d'AS sur une fouille archéologique en Iran.

\section{Traduction de l'article ci-dessus}

1. Un ouvrier manie vigoureusement la pelle. Il est vêtu à l'européenne, conformément aux prescriptions du gouvernement persan, mais il remonte très haut son pantalon une seule jambe, d'ailleurs - pour ne pas être gêné dans ses mouvements.

2. Ce jeune ouvrier est originaire du Sud, comme le révèle son teint basané. Les traits de son visage ont quelque chose de négroïde. C'est l'un de nos chanteurs. Au rythme du travail, il chante volontiers un refrain monotone que les autres ouvriers répètent en chœur.

3. Un ouvrier non qualifié : il n'a rien d'autre à faire que de remonter le panier rempli de la terre que son collègue déblaie. Il utilise pour cela le dispositif primitif que les paysans persans emploient depuis des siècles pour déblayer leurs canaux.

4. Le géomètre effectue quotidiennement un relevé topographique de la fouille.

5. Voici à quoi ressemble une fouille : les cordes blanches servent à baliser des sections de dix mètres carrés par exemple, répertoriées sur le plan par des lettres ou des chiffres. On distingue tout au fond un pan d'un ancien mur de terre battue. Mais les fouilleurs sont déjà parvenus à un niveau plus profond, c'est-à-dire à une "couche " plus ancienne. Les murs des bâtiments qu'on est en train de dégager se trouvent sous le niveau actuel du sol - comme des caves. Ils ont été enfouis sous les débris de bâtiments plus récents - sous la poussière des siècles. - À gauche, on voit un instrument de mesure topographique. La tente au fond ne sert pas d'abri aux fouilleurs; c'est là qu'on entrepose les instruments et les plans, on y prend aussi le petit déjeuner à l'ombre - et c'est là que s'abritent la nuit les veilleurs, indispensables sur toute fouille à cause des "pilleurs de tombes". Aux assistants revient le travail le plus fastidieux : répertorier chaque objet et noter l'endroit et la profondeur où il a été trouvé ; c'est important pour déterminer son âge et la couche de civilisation à laquelle il appartient. Il faut également 
noter quel ouvrier a découvert l'objet en question, car pour éviter que les ouvriers soient tentés de subtiliser des objets pour les revendre plus tard au bazar, on les récompense en leur donnant un «bakchich » approprié.

6. Un autre personnage important sur le chantier de fouille : le jeune porteur d'eau, qui, aux heures les plus chaudes, apporte de l'eau dans un vieux bidon d'essence. Il n'a pas plus de quatorze ans et il est extrêmement fier du maigre salaire qui lui est versé tous les quinze jours.

De nos jours, l'archéologie a beaucoup de points communs avec la quête de l'or et la chasse au trésor. Ce que conteste, bien sûr, tout scientifique rigoureux : il ne fouille pas à cause des objets exhumés, ni à cause de l'or des tombes royales d'Ur ou des précieuses faïences de Samarra - mais pour les résultats, pour l'histoire des civilisations, pour la recherche pure. C'est sans doute avec de tels arguments que se consolent les directeurs de fouilles qui, après une pénible campagne dans la poussière du désert ou dans le paysage désolé de hauts plateaux, doivent abandonner la moitié de leurs découvertes au gouvernement de Syrie, d'Irak ou de Perse, et qui ne rapportent chez eux que des photographies, des plans et des comptes rendus scientifiques.

C'est ainsi qu'ils se consolent. En effet, les fouilleurs - même s'il y a parmi eux quelques idéalistes à tous crins - sont une corporation de gens ayant dans le sang le goût de l'aventure et de la chasse au trésor. Et pas seulement les fouilleurs. Il y a aussi ceux qui mettent sur pied et financent une expédition - tout en restant chez eux - et qui ne sont pas non plus, cela va de soi, totalement désintéressés. Car la question se pose de savoir qui paie ces expéditions archéologiques qui engloutissent des sommes énormes, et dans quel but.

Une fouille est presque toujours financée par un musée ou par le département d'archéologie d'une université. Dans le premier cas, cela dépend naturellement avant tout des découvertes dont on espère qu'elles viendront enrichir le musée et rentabiliseront dans une certaine mesure le capital investi. En même temps, le directeur de la fouille se voit offrir la possibilité de publier un article et de se faire un nom dans la communauté scientifique. Si c'est un institut qui organise l'expédition, les objets exhumés aussi bien que les informations scientifiques récoltées servent la gloire et la réputation de cet institut. Autrefois, on faisait des fouilles avec des moyens financiers relativement limités, les chercheurs vivaient souvent dans des conditions précaires, et en dehors d'un cercle étroit de spécialistes, le monde s'intéressait peu aux résultats. Avec son sens de la propagande et de la publicité, l'Amérique a provoqué un tournant. Étant donné la concurrence des universités, des musées et des instituts, les choses dépendent tout autant de brillants professeurs de réputation internationale que de la richesse, d'une brillante présentation extérieure, de résultats visibles. Des sommes énormes ont été investies pour équiper des expéditions dont on espérait qu'elles allaient découvrir des temples immenses, des palais, des bijoux en or et des statues royales : la tombe de Toutânkhamon, les tombes royales d'Ur et les palais de Persépolis ont rendu l'archéologie populaire; elle suscite aujourd'hui en Amérique autant d'intérêt que le football et les vols transocéaniques. Il est désormais à la mode pour les rois de l'industrie et leurs veuves d'offrir de grosses sommes d'argent aux instituts scientifiques et de parrainer une expédition. Ils viennent visiter les fouilles en avion 
spécial, et leurs noms figurent sur les vitrines des musées où sont exposés les objets exhumés.

Certains archéologues se plaignent non sans raison de cette mode absurde: nombre d'instituts publient des bulletins annuels où les photos des bâtiments des expéditions et de leurs équipements modernes tiennent plus de place que le compte rendu scientifique proprement dit. Mais il faut aussi considérer que ce compromis entre intérêt matériel et intérêt scientifique est nécessaire dans un domaine qui serait condamné à mort sans soutien financier. Même le meilleur des chercheurs ne peut rien faire s'il n'a pas d'argent pour payer des ouvriers, des instruments et tout le matériel allant des pelles et des pioches jusqu'aux caisses et aux sachets en papier - à quoi s'ajoute la concession payée par l'État, le terrain qu'il faut acheter au propriétaire : c'est une petite fortune qui est engagée dans une telle fouille!

Le «camp» est organisé comme un État autonome: le chef de l'expédition et ses assistants en constituent le gouvernement, ils ont à leur disposition leurs propres artisans : menuisier, maçon, cuisinier, veilleur de nuit, chauffeurs, ainsi que la foule des ouvriers, dont le nombre peut varier de 25 à 400 , en fonction de l'étendue et de la richesse de la fouille. Parmi eux, on distingue encore une hiérarchie qui se traduit dans les salaires : qualifiés et non qualifiés, spécialistes et simples "porteurs de paniers ", hommes âgés et expérimentés qui ont déjà participé à de nombreuses campagnes de fouille, et novices incapables de faire la distinction entre un mur d'argile effondré et le sol lui-même. À eux tous, ils forment "l'expédition » typique, dont la vie, même si elle n'est pas toujours aussi romantique que le profane se l'imagine, exerce cependant une fascination très particulière. Celui qui a déjà travaillé « là-bas » reviendra toujours sur les fouilles, malgré les privations et la solitude.

\section{NOTES}

1. Cf. les Lettres à Claude Bourdet, éditions Zoé, Genève 2008, p. 38 et 39.

2. Ibid., p. 7.

3. Mentionné dans le roman Le Refuge des cimes, écrit en 1933.

4. « Ici, le destin est trop près ", lettre à C. Bourdet d'avril 1933, p. 25.

5. Lettre du 3 avril 1934.

6. La Vallée Heureuse, p. 27.

7. La Vallée Heureuse, p. 58.

8. Cf. par exemple à propos du site de Kalneh, la lettre à C. Bourdet du 20 décembre 1933, p. 50 ; sur Baalbek, Hiver au Proche-Orient, p. 80 sq. Et, sur les fouilles à Rihanie, les p. 54 ss. de La Vallée Heureuse.

9. Ella Maillart fit le récit de ce voyage dans La Voie cruelle, Payot, Paris 1988. AS y apparaît sous le pseudonyme « Christina ».

10. Le nom de l'orientaliste et archéologue Joseph Hackin (1886-1941) et celui de sa femme Ria, qui fut sa collaboratrice, sont surtout attachés à la découverte sur le site de Bagram d'un trésor d'objets précieux témoignant de la prospérité de l'Afghanistan sous l'empire kouchan (III ${ }^{\mathrm{e}}$ siècle de notre ère). 
11. Claude Achille Clarac rejoignit les Forces françaises à Alger en décembre 1942, après quoi le général de Gaulle le nomma chef de la mission française à Lisbonne (1943), puis Deuxième conseiller à Tchong-King (1944-45).

12. La Vallée Heureuse, p. 74.

\section{AUTEURS}

DOMINIQUE LAURE MIERMONT

germaniste, traductrice et biographe d'AS

présidente de l'association les Amis d'Annemarie Schwarzenbach

(www.annemarieschwarzenbach.eu)

NICOLE LE BRIS

normalienne, agrégée de lettres classiques, néo-helléniste, traductrice nicolelebris@yahoo.fr 